\title{
Comorbidity of psychiatric disorders and symmetric distal polyneuropathy among type II diabetic outpatients
}

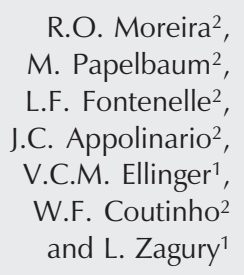

R.O. Moreira², M. Papelbaum ${ }^{2}$, L.F. Fontenelle ${ }^{2}$, J.C. Appolinario ${ }^{2}$, V.C.M. Ellinger ${ }^{1}$, W.F. Coutinho ${ }^{2}$ and L. Zagury ${ }^{1}$

\author{
'Serviço de Diabetes, Instituto Estadual de Diabetes e Endocrinologia, \\ ${ }^{2}$ Unidade de Psiconeuroendocrinologia, \\ Instituto Estadual de Diabetes e Endocrinologia/Instituto de Psiquiatria, \\ Universidade Federal do Rio de Janeiro, Rio de Janeiro, RJ, Brasil
}

\section{Correspondence}

R.O. Moreira

Instituto Estadual de Diabetes

e Endocrinologia

Rua Moncorvo Filho, 90

20211-340 Rio de Janeiro, RJ

Brasil

Fax: +55-21-2224-9562

E-mail: rom_br@yahoo.com

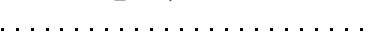

Received September 20, 2005

Accepted October 24, 2006

\begin{abstract}
The objective of the present study was to establish the frequency of psychiatric comorbidity in a sample of diabetic patients with symmetric distal polyneuropathy (SDPN). Sixty-five patients with type 2 diabetes mellitus were selected consecutively to participate in the study at Instituto Estadual de Diabetes e Endocrinologia. All patients were submitted to a complete clinical and psychiatric evaluation, including the Portuguese version of the structured clinical interview for DSM-IV, the Beck Depression Inventory, the Neuropathy Symptom Score, and Neuropathy Disability Score. SDPN was identified in 22 subjects (33.8\%). Patients with and without SDPN did not differ significantly regarding sociodemographic characteristics. However, a trend toward a worse glycemic control was found in patients with SDPN in comparison to patients without SDPN $\left(\mathrm{HbA}_{1 \mathrm{c}}=8.43 \pm 1.97\right.$ vs $7.48 \pm 1.95 ; \mathrm{P}=0.08$ ). Patients with SDPN exhibited axis I psychiatric disorders significantly more often than those without SDPN (especially anxiety disorders, in general (81.8 vs 60.0\%; $\mathrm{P}=$ 0.01 ), and major depression - current episode, in particular (18.2 vs $7.7 \% ; \mathrm{P}=0.04)$ ). The severity of the depressive symptoms correlated positively with the severity of SDPN symptoms $(r=0.38 ; P=0.006)$, but not with the severity of SDPN signs $(r=0.07 ; \mathrm{P}=0.56)$. In conclusion, the presence of SDPN seems to be associated with a trend toward glycemic control. The diagnosis of SDPN in diabetic subjects seems also to be associated with relevant psychiatric comorbidity, including anxiety and current mood disorders.
\end{abstract}

Diabetic neuropathy is one of the most common microvascular complications of diabetes mellitus (DM). It is characterized by progressive neural impairment affecting both peripheral nerves and the central nervous system (1). Symmetric distal polyneuropa-
Key words

- Anxiety

- Depressive symptoms

- Diabetic neuropathy

- Symmetric distal

polyneuropathy

- Psychiatric interview

- Psychometric scales thy (SDPN) is considered to be the most frequent presentation of diabetic neuropathy (1). Patients with SDPN often complain of acute, sub-acute or chronic pain, especially in the foot and with a characteristic night exacerbation. Along with these symptoms, 
patients with SDPN can also display sensory and motor impairment leading from an altered thermic, painful, and vibratory sensitivity in the foot to the complete absence of deep tendon reflexes (2). Furthermore, it is important to mention that the clinical presentation of SDPN may vary widely from undetectable to incapacitating symptoms and/ or signs.

It has been reported that individuals with DM, particularly those suffering from diabetic complications (such as retinopathy and nephropathy), may be more prone to develop a certain set of psychiatric disorders, especially mood disorders (3). It is still not known, however, whether diabetic patients with SDPN may also exhibit increased rates of mood and/or anxiety disorders. The lack of objectiveness in the diagnosis of SDPN associated with a large number of diagnostic instruments available for the assessment of psychiatric symptoms may be partially responsible for the heterogeneity of the current findings.

In the present study, we intended to determine the frequency of psychiatric comorbidity in diabetic patients with SDPN and compare it to diabetic patients without SDPN using reliable and well-defined methods. We also investigated the relationship between depressive symptoms and signs/symptoms of SDPN.

Sixty-five type 2 diabetic outpatients aged 30 to 65 years were selected consecutively to participate in the study at the Serviço de Diabetes of Instituto Estadual de Diabetes e Endocrinologia from January 2003 to December 2003. Type 2 DM was diagnosed according to American Diabetes Association criteria (4). All participants were examined by an experienced endocrinologist (ROM) and provided a detailed medical history at baseline evaluation.

The protocol was approved by the Ethics Committee of the Institution and written informed consent was obtained from each patient after the procedures involved in the study were fully explained. Patients were excluded if they had any medical diseases or neurologic disorders that could be associated with neuropathy or if they were under psychiatric treatment. Based on these criteria, 3 participants were excluded (2 with a history of alcohol abuse and 1 with Hansen's disease). Patients were also excluded if they were undergoing dialysis or if they were unable to read.

Initially, all participants were examined by one of the authors (ROM or MP) using a sociodemographic questionnaire. The educational level was defined as the total number of years of formal education completed by each patient. Marital status was defined as either married (cohabiting) or unmarried (living unaccompanied). Family income was defined as the number of Brazilian minimum wages, which correspond to US $\$ 100$ to US\$125.

The following anthropometric data were recorded for all participants: body weight $(\mathrm{kg})$, height $(\mathrm{m})$, body mass index, waist circumference, and waist-hip ratio. Body mass index was calculated as weight in kilograms divided by the square of height in meters $\left(\mathrm{kg} / \mathrm{m}^{2}\right)$. Waist circumference was determined at the midpoint between the lowest rib and the iliac crest. Waist-hip ratio was defined as the ratio of waist girth to the largest circumference of the hips, measured at the trochanter major.

For the assessment of neuropathy, Portuguese versions of the Neuropathy Disability Score (NDS) and Neuropathy Symptom Score (NSS) were used by two independent raters (ROM and LFF). The final score was the mean value of their evaluations. NSS and NDS are the most common instruments used for the assessment of SDPN in clinical research. NSS and NDS have already been adequately translated into Portuguese and are both considered to be reliable instruments (5). Moreover, they cover both symptoms and signs of SDPN. The NDS and NSS versions used in this study were derived 
from the version modified by Young et al. (6).

NDS was obtained with the examination of the ankle reflex, vibration, pin-prick, and temperature sensation (cold tuning fork) in the big toe. The total maximum abnormal score on this scale was 10 . A score of 3-5 was regarded as evidence of mild neuropathic signs, 6-8 as evidence of moderate signs and a score of 9-10 was regarded as evidence of severe signs of neuropathy. The NSS was based on questioning the patients about their experiences of pain or discomfort in the legs. The maximum symptom score on this scale was 9. A symptom score of 3-4 was regarded as mild symptoms, a score of 5-6 as moderate symptoms and a score of 7-9 as severe symptoms of neuropathy. The minimum acceptable criteria for a diagnosis of peripheral neuropathy were: moderate signs with or without symptoms, or mild signs with moderate symptoms. Mild signs alone or with mild symptoms were not considered to be an adequate parameter for a diagnosis of peripheral neuropathy.

The prevalence of psychiatric disorders in patients with type $2 \mathrm{DM}$ was assessed using the Portuguese version of the Structured Clinical Interview for DSM-IV/Patient version (SCID-I/P - Axis I Disorder) (7). This version has already been validated in a Brazilian population (8). All diagnostic interviews were conducted by a trained psychiatrist (MP), who was blind to the neurological status of the patients and who had long experience in the evaluation of psychiatric disorders among patients with DM.

The Beck Depression Inventory (BDI) is a 21-item self-report rating inventory measuring characteristic attitudes and symptoms of depression (9). Although it evaluates the physical symptoms of depression (besides the cognitive ones), the BDI has been shown to be reliable for the investigation of depression in diabetic patients (10). The Portuguese version of the BDI used in this study has been validated in non-clinical popula- tions, but not in diabetic ones (11).

Venous blood samples were collected after a 12-h fast. Blood glucose was measured by a glucose oxidase method and glycosylated hemoglobin $\left(\mathrm{HbA}_{\mathrm{lc}}\right)$ concentrations were measured by high-pressure liquid chromatography. The reference values for the assay were 4-6\%.

Statistical analysis was performed with the GraphPad InStat 3.00 for Windows 95 software (GraphPad Software, San Diego, CA, USA). Diabetic patients with SDPN were compared and contrasted with individuals with DM without SDPN using two tailed $t$-tests to analyze continuous variables and Fisher's exact test to study categorical data (Table 1). Fisher's exact test was employed to compare the frequency of a psychiatric diagnosis according to the presence of SDPN (Table 2). The strength of the linear relationship between two continuous variables (i.e., BDI and NDS or NSS scores) was evaluated by the Pearson test for parametric variables. The level of statistical significance was set at $5 \%$.

Table 1. Characteristics of diabetic patients with and without symmetric distal polyneuropathy (SDPN).

\begin{tabular}{lcc}
\hline & $\begin{array}{c}\text { With SDPN } \\
(\mathrm{N}=22)\end{array}$ & $\begin{array}{c}\text { Without SDPN } \\
(\mathrm{N}=43)\end{array}$ \\
\hline Gender (male/female) & $4 / 18$ & $8 / 35$ \\
Age (years) & $52.8 \pm 5.5$ & $53.0 \pm 7.3$ \\
Body mass index $\left(\mathrm{kg} / \mathrm{m}^{2}\right)$ & $32.1 \pm 5.3$ & $29.9 \pm 4.8$ \\
Waist/hip ratio & $0.92 \pm 0.05$ & $0.92 \pm 0.11$ \\
Education & & \\
1-8 years & 16 & 29 \\
$9-12$ years & 6 & 9 \\
$>13$ years & 0 & 3 \\
Income (minimum wage) & $5.2 \pm 3.9$ & $72.7 \%$ \\
Marital status (married) & $17.7 \pm 5.7 \%$ & $10.7 \pm 5.1^{*}$ \\
Duration of diabetes (years) & $195.0 \pm 80.8$ & $150.0 \pm 81.9^{*}$ \\
Glucose (mg/dL) & $8.43 \pm 1.97$ & $7.48 \pm 1.95$ \\
Glycosylated hemoglobin (\%) & $4.6 \pm 2.4$ & $1.6 \pm 1.5^{*}$ \\
Neuropathic Disability Score & $6.0 \pm 1.9$ & $2.3 \pm 2.0^{*}$ \\
Neuropathic Symptom Score & $13.9 \pm 10.0$ & $17.8 \pm 7.5$ \\
Beck Depression Inventory & & \\
\hline Data are reported as means \pm SD, & number of patients (gender and education) and \\
percent (marital status). & & \\
*P < 0.05 compared to patients with SDPN (unpaired $t$-test). &
\end{tabular}


SDPN was identified in 22 subjects (33.8\%). No significant differences between patients with and without SDPN were found with regard to sociodemographic characteristics (Table 1). In relation to neuropathic signs and symptoms, diabetic patients with SDPN exhibited higher scores in NSS and NDS ( $\mathrm{P}<0.001$ for both scores). Glycemic control was evaluated using fasting glucose and $\mathrm{HbA}_{1 \mathrm{c}}$ levels. Although the presence of SDPN was associated with higher levels of fasting glucose $(P=0.049)$, only a trend toward significance was observed with $\mathrm{HbA}_{1 \mathrm{c}}$ levels $(P=0.08)$. Diabetic patients with SDPN exhibited axis I psychiatric disorders significantly more often than patients without SDPN, especially anxiety disorders. Among mood disorders, major depressive disorder - current episode, in particular -, was found to be significantly more frequent in patients with SDPN than in their nonSDPN counterparts (Table 2). An additional analysis of the association between SDPN and psychiatric comorbidity was performed using NDS and NSS independently. With this procedure no relationship was found between the severity of either SDPN signs or symptoms and psychiatric comorbidity $(\mathrm{P}=$ 0.63 and $\mathrm{P}=0.56$, respectively). However,

\begin{tabular}{|c|c|c|c|}
\hline & $\begin{array}{l}\text { Total sample } \\
\qquad(\mathrm{N}=65)\end{array}$ & $\begin{array}{l}\text { With SDPN } \\
\qquad(\mathrm{N}=22)\end{array}$ & $\begin{array}{l}\text { Without SDPN } \\
\quad(N=43)\end{array}$ \\
\hline Any Axis I disorder & $38(58.4 \%)$ & $20(90.9 \%)$ & $18(41.8 \%)^{\star}$ \\
\hline Any current mood disorder & $14(21.5 \%)$ & $9(40.9 \%)$ & $5(11.6 \%)^{*}$ \\
\hline Major depressive disorder & $5(7.7 \%)$ & $4(18.2 \%)$ & $1(2.3 \%)^{*}$ \\
\hline Bipolar disorder & $3(4.6 \%)$ & $1(4.5 \%)$ & $2(4.6 \%)$ \\
\hline Dysthymic disorder & $7(10.7 \%)$ & $4(18.2 \%)$ & $3(7.0 \%)$ \\
\hline Any anxiety disorder & $39(60.0 \%)$ & $18(81.8 \%)$ & $21(48.8 \%)^{*}$ \\
\hline Agoraphobia & $3(4.6 \%)$ & $1(4.5 \%)$ & $2(4.6 \%)$ \\
\hline Panic disorder without agoraphobia & $6(17.1 \%)$ & $3(12.5 \%)$ & $3(6.9 \%)$ \\
\hline Specific phobia & 15 (23.0\%) & 8 (36.4\%) & $7(16.2 \%)$ \\
\hline Social phobia & $16(24.6 \%)$ & 7 (31.8\%) & 9 (20.9\%) \\
\hline Generalized anxiety disorder & $16(24.6 \%)$ & 7 (31.8\%) & 9 (20.9\%) \\
\hline Anxiety disorder not otherwise specified & $6(17.1 \%)$ & $4(18.1 \%)$ & $2(4.6 \%)$ \\
\hline
\end{tabular}

Data are reported as number of patients with percent in parentheses. ${ }^{*} \mathrm{P}<0.05$ compared to patients with SDPN (Fischer exact test). the severity of the depressive symptoms (BDI) correlated positively with the severity of the SDPN symptoms (NSS; $r=0.38 ; \mathrm{P}=$ 0.006 ), but not with the severity of the SDPN signs (NDS; $r=0.07 ; \mathrm{P}=0.56$ ).

We determined whether patients with type 2 DM and SDPN are characterized by increased rates of comorbid psychiatric disorders in comparison with patients with DM and without SDPN. To this end, we systematically evaluated the sociodemographic features, the anthropometric characteristics, the neurological findings, and the mental status of 65 patients with type $2 \mathrm{DM}$. Our most significant findings were the following: 1) individuals with type 2 DM and SDPN exhibited axis I psychiatric disorders significantly more often than those without SDPN (especially anxiety disorders, in general, and a current episode of major depression, in particular), and 2) the severity of the depressive symptoms correlated positively with the severity of the neuropathic symptoms.

Our findings suggest the presence of a significant link between SDPN and the presence of a current mood disorder, especially major depression. Several authors have already investigated the relationship between diabetic neuropathy and depression (for a review, see Ref. 12). These investigations, in contrast to our study, were characterized by diverse and/or inconsistent approaches to the assessment of diabetic neuropathy (including nerve conduction studies in some of them and unreliable assessment of signs and/ or symptoms in others). Moreover, all studies that managed to demonstrate the existence of a relationship were based exclusively on self-report instruments (Zung's scale, BDI, and KDS-1) (see Ref. 12). This heterogeneity of methods may affect the results of a meta-analysis published by de Groot et al. (12), which demonstrated that neuropathy was the diabetic complication with the weaker evidence for a relationship with depression. We believe that one of the strengths of the present study was the adop- 
tion of more valid and reliable instruments (i.e., the SCID, the NDS and the NSS) which were employed by experienced clinicians.

Although we were able to identify a close relationship between the presence of anxiety disorders and SDPN in type 2 DM patients, it is not clear, at the present moment, which condition may be "primary" on chronological grounds. Some individuals with SDPN may develop anxiety disorders as a complication of their chronic medical condition. For example, it is conceivable that having type $2 \mathrm{DM}$ with complications may have filled the mental images of our patients with worries and concerns about health issues. Alternatively, other patients suffering from anxiety disorders may be more prone to overestimating some physical sensations (including neuropathy-like symptoms) that would otherwise be undetectable. Indeed, the overestimation of physical symptoms was demonstrated in other anxiety disorders and may have contributed to their development in some etiological models (13).

The BDI remains an important instrument in the evaluation of depressive symptoms in diabetic patients. In patients with SDPN symptoms, however, its results should be carefully interpreted, since there may exist an overlapping between neuropathic and depressive symptoms leading to falsepositive results. A plausible explanation for this finding is the fact that one of the most common complaints related to SDPN is pain. The presence of chronic pain has been associated with the presence and severity of mood disorders (14). It is also interesting to note that the presence of depressive symptoms has been associated with the development of several types of pain, without any reasonable explanation for these feelings (15). On the other hand, one could argue that some of the patients in the present study may have been unable to differentiate between depressive and neuropathic symptoms. These findings would suggest that complaints suggestive of SDPN should be taken with caution and in fact may be consistent with the presence of depressive symptoms. An experienced psychiatrist, especially by using the SCID, would be more capable of correctly "dissecting" these conditions from one another. Indeed, this was supported by our finding, i.e., a significant association was found between a current clinical evaluation of the mood disorders and SDPN.

The relationship among glycemic control, microvascular complications (i.e., neuropathy) and psychiatric comorbidity is another relevant finding of our study. It is widely accepted that a poor glycemic control is associated with a progressive worsening in nerve damage, leading to SDPN (16). The higher levels of plasma glucose and $\mathrm{HbA}_{1 \mathrm{c}}$ found in diabetic patients with SDPN support these findings. Also, depression has already been associated with a poor glycemic control and diabetic neuropathy (12). In line with this, the trend toward a worse glycemic control observed in our patients with SDPN may be attributed to uncontrolled $\mathrm{DM}$ or to an undiagnosed psychiatric disorder, or, more likely, to both. Therefore, the presence of a psychiatric disorder in a diabetic patient with neuropathy can be considered either a cause or a consequence of unsatisfactory glycemic control and of microvascular complications.

The present study has some limitations. Perhaps one of the most significant lies in the scope of psychiatric and medical assessment. Future studies should assess the presence of milder forms of psychopathology and systematically evaluate patients for the presence of the whole spectrum of complications associated with type 2 DM. For example, depressive symptoms in subjects with diabetes-related complications (e.g., lower sex-drive) may be present in patients more because of impotence problems than because of SDPN. Widening the scope of medical assessment would help disentangle the role played by each of the diabetic complications in the mental health of our patients. 
Second, our sample size may limit the strength of our findings. Although we were able to demonstrate a statistically significant association between psychiatric syndromes and diabetic neuropathy, the small number of diabetic patients with a psychiatric diagnosis (especially depression) should be carefully interpreted and may not reflect the real prevalence of this comorbidity. Third, there are other methods that can be used in the evaluation of SDPN. The use of a visual analogue scale to determine the severity of the neuropathic pain or electroneuromyography to determine the severity and type of nerve damage may bring new insights into the study of the relationship between SDPN and psychiatric disorders. Fourth, neither the presence of nephropathy nor the presence of retinopathy was evaluated in this study. Since both complications can co-exist with neuropathy, we could not differentiate which one exerts the most significant impact on depressive symptomatology. Finally, prospective studies are necessary to clarify the impact of the progressive nerve lesion on diabetic neuropathy and the development of specific psychiatric disorders, especially mood and anxiety disorders.

Nevertheless, the presence of "milder" forms of mood disorders, such as minor depressive disorder or mixed anxiety-depressive disorder, was not covered by our assessment. In fact, increasing evidence suggests that patients with chronic medical con- ditions may be more prone to developing such disorders $(17,18)$. Although a direct relationship between mood disorders and DM has only been demonstrated in patients with major depression (19), we believe that milder forms of mood disorders may be a common finding in some diabetic patients, especially those with a diagnosis dating back a long time. The treatment of DM involves many lifestyle modifications. These modifications, most of the time restrictions, however, are not always well accepted by some diabetic patients. These patients, in particular, represent the majority of our population and may be more prone to mild forms of psychiatric comorbidity.

Diabetic patients with SDPN seem to exhibit increased rates of psychiatric diseases in comparison to diabetic patients without SDPN, particularly anxiety and depression. Moreover, a direct relationship between neuropathic and depressive symptoms was also demonstrated. The use of self-rating scales in the evaluation of depression or neuropathy in diabetic patients should be cautiously interpreted, since the overlapping of both symptoms might lead to an overestimation of their symptoms by the patient. Therefore, the evaluation of depressive symptoms in a diabetic patient with diabetic neuropathy should be done by a trained psychiatrist, who will be able to adequately identify depressive symptomatology.

\section{References}

1. Boulton AJ, Gries FA, Jervell JA. Guidelines for the diagnosis and outpatient management of diabetic peripheral neuropathy. Diabet Med 1998; 15: 508-514.

2. Partanen J, Niskanen L, Lehtinen J, Mervaala E, Siitonen O, Uusitupa M. Natural history of peripheral neuropathy in patients with non-insulin-dependent diabetes mellitus. N Engl J Med 1995; 333: 89-94.

3. Lloyd CE, Dyer PH, Barnett AH. Prevalence of symptoms of depression and anxiety in a diabetes clinic population. Diabet Med 2000; 17: 198-202.

4. Report of the Expert Committee on the Diagnosis and Classification of Diabetes Mellitus. Diabetes Care 1997; 20: 1183-1197.

5. Moreira RO, Castro AP, Papelbaum M, Appolinario JC, Ellinger VC, Coutinho WF, et al. Translation into Portuguese and assessment of the reliability of a scale for the diagnosis of diabetic distal polyneuropathy. Arq Bras Endocrinol Metabol 2005; 49: 944-950.

6. Young MJ, Boulton AJ, MacLeod AF, Williams DR, Sonksen PH. A multicentre study of the prevalence of diabetic peripheral neuropathy in the United Kingdom hospital clinic population. Diabetologia 1993; 36: 150-154.

7. First MB, Spitzer RL, Gibbon M, Williams JB. Structured clinical interview for DSM-IV-TR axis I disorders, research version, patient 
edition (SCID-I/P). In: Anonymous, Biometrics research. New York: New York State Psychiatric Institute; 2002.

8. Del-Ben CM, Rodrigues CR, Zuardi AW. Reliability of the Portuguese version of the structured clinical interview for DSM-III-R (SCID) in a Brazilian sample of psychiatric outpatients. Braz J Med Biol Res 1996; 29: 1675-1682.

9. Beck AT, Ward CH, Mendelson M, Mock J, Erbaugh J. An inventory for measuring depression. Arch Gen Psychiatry 1961; 4: 561-571.

10. Lustman PJ, Clouse RE, Griffith LS, Carney RM, Freedland KE. Screening for depression in diabetes using the Beck Depression Inventory. Psychosom Med 1997; 59: 24-31.

11. Gorenstein C, Andrade L, Vieira Filho AH, Tung TC, Artes R. Psychometric properties of the Portuguese version of the Beck Depression Inventory on Brazilian college students. J Clin Psychol 1999; 55: 553-562.

12. de Groot M, Anderson R, Freedland KE, Clouse RE, Lustman PJ. Association of depression and diabetes complications: a meta-analysis. Psychosom Med 2001; 63: 619-630.
13. Lautenbacher S, Krieg JC. Pain perception in psychiatric disorders: a review of the literature. J Psychiatr Res 1994; 28: 109-122.

14. Clark MR, Treisman GJ. Perspectives on pain and depression. Adv Psychosom Med 2004; 25: 1-27.

15. Lepine JP, Briley M. The epidemiology of pain in depression. Hum Psychopharmacol 2004; 19 (Suppl 1): S3-S7.

16. The Diabetes Control and Complications Trial Research Group. The effect of intensive diabetes therapy on the development and progression of neuropathy. Ann Intern Med 1995; 122: 561-568.

17. Fraguas R Jr, Alves TC. Depression in General Hospital: a study of 136 cases. Rev Assoc Med Bras 2002; 48: 225-230.

18. Wittchen HU, Essau CA. Comorbidity and mixed anxiety-depressive disorders: is there epidemiologic evidence? J Clin Psychiatry 1993; 54 (Suppl): 9-15.

19. Eaton WW, Armenian H, Gallo J, Pratt L, Ford DE. Depression and risk for onset of type II diabetes. A prospective population-based study. Diabetes Care 1996; 19: 1097-1102. 